\title{
Conduction mechanism in anthracene as a function of temperature
}

\author{
JEENA V JOSEPH and FRANCIS P XAVIER* \\ Physics Department, Loyola Institute of Frontier Energy (LIFE), Loyola College, Chennai \\ 600034, India \\ MS received 2 March 1996; revised 15 April 1997

\begin{abstract}
Conduction mechanism in anthracene single crystal grown by Bridgman method was carried out. The investigations consisted of dark- and photo-current variation with respect to (i) applied electric field and (ii) temperature. The applied electric field ranged from 0.5 to $2.5 \mathrm{kV} / \mathrm{cm}$ and the temperature range was between $300 \mathrm{~K}$ and $450 \mathrm{~K}$. Photo and dark current variations with temperature indicate, based on activation energy determination, that a band model can be applied to the conduction process. The band gap is calculated to be $1.6 \mathrm{eV}$. The band model consists of a recombination centre $0.37 \mathrm{eV}$ above the valence band edge and a trap level $0.55 \mathrm{eV}$ below the conduction band edge to which electrons are first thermo-optically excited and then they are thermally excited into the conduction band.
\end{abstract}

Keywords. Anthracene; photoconductivity; band gap.

\section{Introduction}

Organic molecules in condensed solid phase are called molecular crystals. Molecular crystals are different from traditional solid state crystals such as covalent or ionic crystals in optical and electronic properties. This is due to weak inter molecular interaction force, called van der Waals' force, with binding energy considerably lower than that of covalent or ionic bonds in atomic crystals (Silinsh 1980). Some organic materials, such as anthracene, containing conjugate bond system (single-double bonding) in their crystal structure are photosensitive. Electrons associated with these bonds are not localized on any particular atom but are associated with the entire molecule (Xavier 1993). These electrons are in the p-state and are hence called pi-electrons. The pi-electrons are believed to be responsible for conduction in organic conductors. When pi-electrons are excited, thermally as well as optically, into free state, holes are left behind on the molecule. It is these holes and electrons which participate in the conduction process. And the transport of charge carriers take place under an applied electric field. Anthracene is one of the extensively studied organic photoconductors which consists of three linear benzene rings for its crystal structure. Anthracene is a monoclinic crystal with the crystal constants as $a=8.56 \AA, b=6.04 \AA, c=11 \cdot 16 \AA$ and $\alpha=90^{\circ}, \beta=124^{\circ}$, and $\gamma=90^{\circ}$ (Silinsh 1980).

Though absorption and reflectance based on spectral response could serve as tools for studying conduction mechanism, especially of band-gap, these spectra, in general, exhibit broad maxima. Since electrical transport investigations indicate relatively sharper maxima corresponding to excitation of charge carriers across the band-gap, these results could be more reliable even in the case of organic semiconductors in spite of possible deviations from conventional semiconductor theories.

*Address for communication 
Anthracene micro crystals may be used as media for optical signal transfer in molecular structures (Aviksoo and Reinot 1992). Anthracene has been used as a model system in a large number of electronic polarization energy calculations (Batley and Lyons 1970) and in studying local trapping centres for excitons and charge carriers (Welfrich and Lipsett 1965). Attempt has been made to calculate the structure of conductivity bands in one-electron approximation (Le Blanc 1962).

\section{Sample preparation}

The anthracene crystal was grown a few years ago by Xue and Goldsmith (1992) using Bridgman method. Since anthracene crystal decomposes before meiting, the maximum temperature gradient applied in the crystal growth process was below the decomposition temperature. The X-ray diffraction of the crystal indicates certain amount of mosaic spread thereby confirming the existence of flakes and grain boundaries in the crystal grown. The crystal constants are also slightly different from the standard reported values. The crystal constants of anthracene crystal used in our investigations are compared with the standard values in table 1 . The difference in crystal constants $c$ and $\beta$ may be due to the fact that the molecules in alternate stacks are wedged together. And this wedging could take place in three ways depending on how the $\mathrm{CH}$ bonds of one fit into the rings of adjacent molecule. The two common ways are shown in figure 1 (Hall 1991).

\section{Experimental}

\subsection{Dark conductivity}

When the material is kept in the dark, the energy bands adjust themselves relative to the Fermi level and reach an equilibrium state. Now if an electric field is applied this equilibrium is disturbed bringing about changes in barrier widths and heights both inside the crystal and at the crystal electrode boundaries. As a result there will be a net flow of charges until a new equilibrium distribution is obtained and this flow of charges is the 'dark' current.

The sample, kept in dark, was connected to a dc power supply and a pico-ammeter (Keithley) in series. After making sure the ohmicity at the electrodes of the sample, the dark current of the sample was measured as a function of temperature. The dark current, $I_{\mathrm{d}}$, varies with temperature, $T$, according to the relation

$$
I_{\mathrm{d}}=I_{0} \exp \{-E / k T\},
$$

Table 1. Comparison of crystal constants of anthracene crystal used in the present investigations with those of standard values.

\begin{tabular}{lcc}
\hline & Standard values & Present values \\
\hline$a \AA$ & 8.56 & 8.54 \\
$b \AA$ & 6.04 & 6.0 \\
$c \AA$ & $11 \cdot 16$ & $9 \cdot 45$ \\
$\alpha$ & $90^{\circ}$ & $90^{\circ}$ \\
$\beta$ & $124^{\circ}$ & $103 \cdot 4^{\circ}$ \\
$\gamma$ & $90^{\circ}$ & $90^{\circ}$ \\
\hline
\end{tabular}



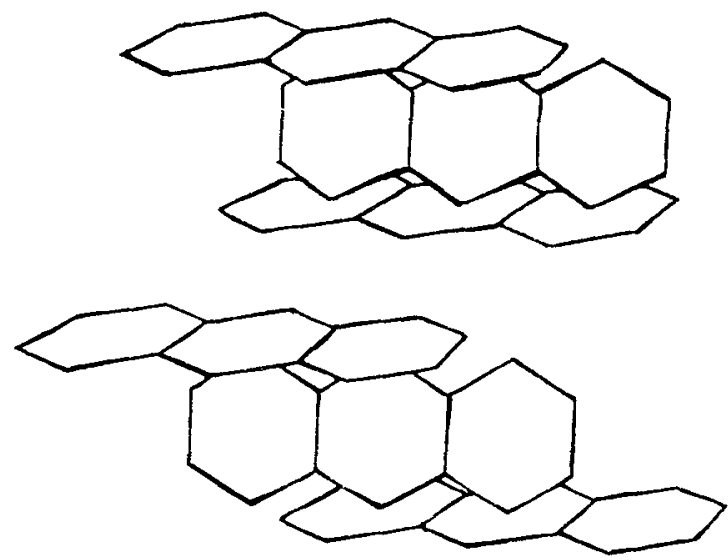

Figure 1. Wedging of anthracene molecules in alternate stacks.

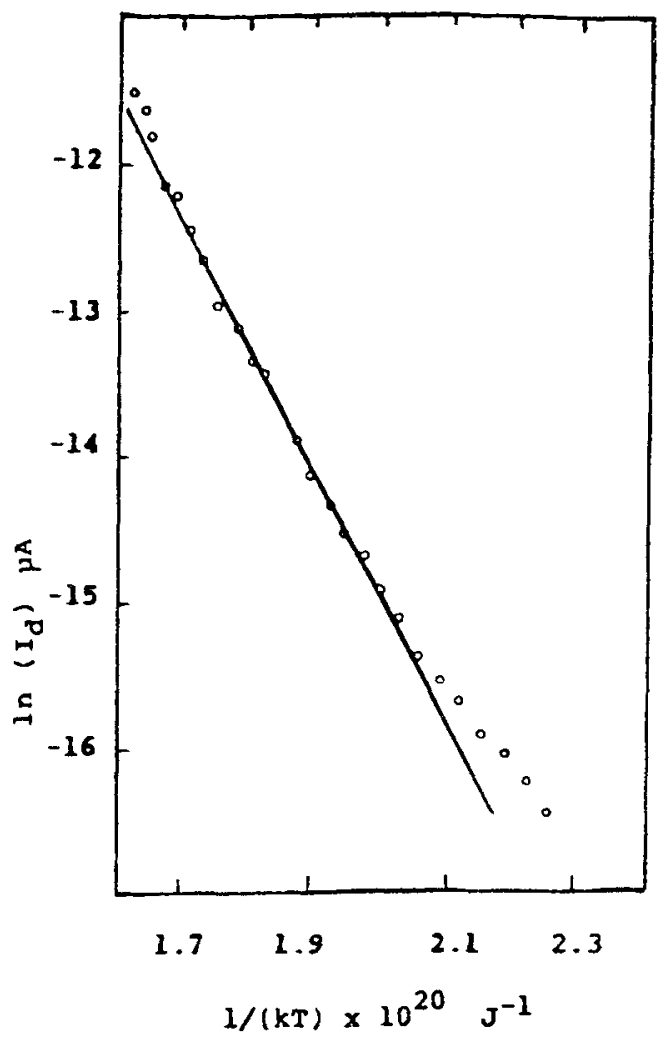

Figure 2. $\ln I_{\mathrm{d}}$ vs $(1 / k T)$ for anthracene crystal.

with $E$, activation energy in $\mathrm{eV}$. The variation of dark current as a function of temperature up to $450 \mathrm{~K}$ was studied and the activation energy was determined to be $0.55 \mathrm{eV}$ from a $\ln I_{\mathrm{d}}$ vs $(1 / k T)$ plot (figure 2$)$. Since the experiment was conducted in the open, the sample got decomposed above $450 \mathrm{~K}$. And from the phenomenological relation (Simon and Andre 1985) of most organic semiconductor, 


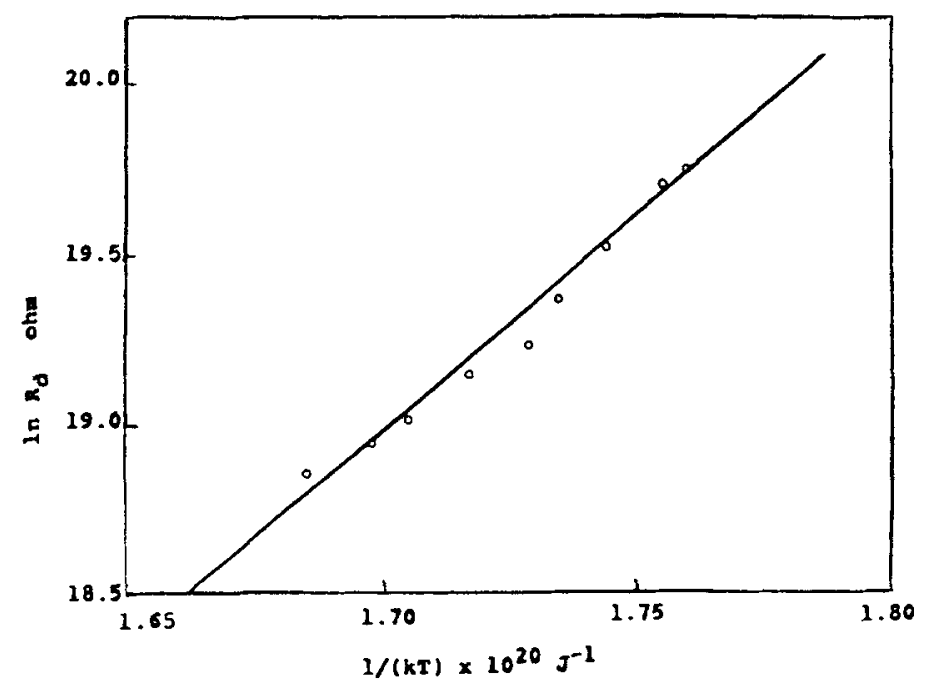

Figure 3. $\ln R$ vs $(1 / k T)$ for anthracene crystal.

due to inhomogeneity in material (Boer 1990), for temperature dependent resistance, $R$, viz.

$$
R=R_{0} \exp \left\{E_{\mathrm{g}} / 2 k T\right\},
$$

with $E_{\mathrm{g}}$, band gap energy of the semiconductor, the band gap of the sample was calculated to be $1.6 \mathrm{eV}$ from the action energy $\left(E=E_{\mathrm{g}} / 2=0.8 \mathrm{eV}\right)$ from slope of plot $\ln R$ vs $(1 / k T)$ as shown in figure 3 .

\subsection{Photoconductivity}

As $\mathrm{He}-\mathrm{Ne}$ laser $(5 \mathrm{~mW})$ was shone on the sample there was increase in current flow across the sample when compared with dark current for the same applied field. The applied potential was increased from 0 to $500 \mathrm{~V}$, across the electrode distance of about $2 \mathrm{~mm}$ and the variation of photocurrent, $I_{\mathrm{ph}}$, as a function of temperature, $T$, was investigated according to the relation

$$
I_{\mathrm{ph}}=I_{0} \exp \{-E / k T\},
$$

with $E$, photo activation energy in $\mathrm{eV}$. A plot of $\ln \left(I_{\mathrm{ph}}\right)$ vs $(1 / k T)$ indicates two slopes with $E_{1}=0.37 \mathrm{eV}$ and $E_{2}=0.68 \mathrm{eV}$ (figure 4), which are taken to be activation energies.

\section{Discussion}

The organic semiconductors are known to have narrow energy band. Further, there exists more than one trap level in the forbidden gap which can cause instantaneous retrapping even in the dark (Mort and Pai 1976). In the present investigation the dark activation energy is found to be $0.55 \mathrm{eV}$ and this can be interpreted that there is a level 


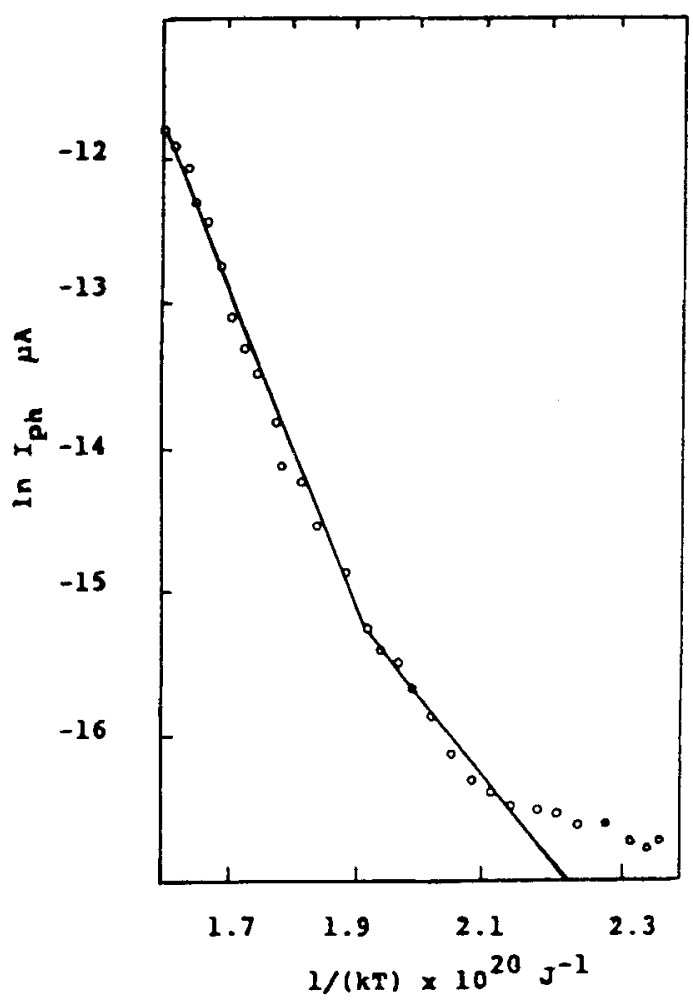

Figure 4. $\ln I_{\mathrm{ph}}$ vs $(1 / k T)$ for anthracene crystal.

$0.55 \mathrm{eV}$ below the conduction band edge (trap level) from which charge carriers could be thermally excited into the conduction band.

General consideration on temperature dependence of charge carrier motion indicates that the conductivity is due to either tunneling or hopping process. When the conductivity is independent of temperature then the conduction process is called tunneling and when the conduction is temperature-dependent, then it is known as hopping process (Xavier 1993). But in the case of molecular crystals tunneling and hopping models of conduction are not exclusive processes. And there might exist experimentally observable transition regions from band-to-hopping motion (Silinsh 1980 ) in organic molecular solids. Based on these assumptions the two photo activation energy levels, viz. $0.37 \mathrm{eV}$ and $0.68 \mathrm{eV}$, could be interpreted as follows: (i) the electrons are excited to an energy level $0.37 \mathrm{eV}$ above the valence band (which is also the recombination centre) by tunneling or hopping and (ii) from this state they are optically excited to a level $0.68 \mathrm{eV}$ above this recombination centre which is called trap level. And the charge carrier from this trap level are also excited thermally into the conduction band. So thermo-optically the electrons could be totally excited to a level $1.05 \mathrm{eV}$ above the valence band edge and from which they are further thermally excited $0.55 \mathrm{eV}$ into the conduction band. Thus the band gap corresponds to the calculated energy of $1.6 \mathrm{eV}$.

The reported band gap values of anthracene vary from $1.5 \mathrm{eV}$ up to $4.4 \mathrm{eV}$ (Gutmann and Lyons 1981). Most of the work done on single crystal report value around $1.6 \mathrm{eV}$, for example, the value of $1.66 \mathrm{eV}$ is reported by Eley et al (1953) and 


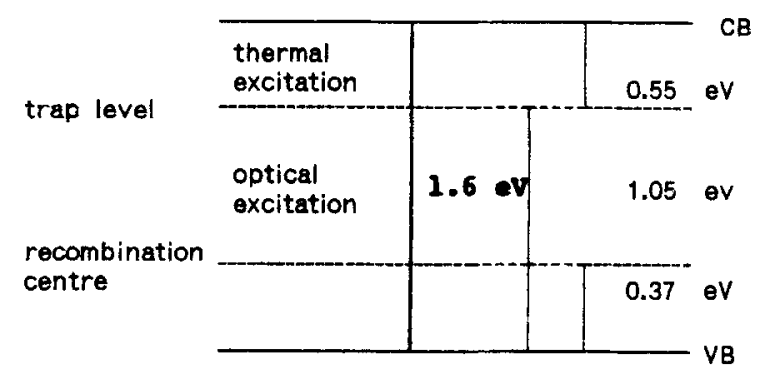

Figure 5. Band model for anthracene crystal.

Mette and Pick (1953) and the value of $1.5 \mathrm{eV}$ is reported by Riehl $(1955,1957)$. The variation in band gap can be ascribed to the difference in wedging of molecules in alternate stacks (figure 1) and the difference in impurity levels in the crystals taken for investigation.

\section{Conclusions}

Xavier and Goldsmith (1995) have indicated that conduction mechanism involves trap level system in organic photoconductors. Based on the experimental observations, viz. dark activation energy of $0.55 \mathrm{eV}$ and photo activation energies of 0.37 and $0.68 \mathrm{eV}$, we can conclude that the band gap of anthracene single crystal is $1.6 \mathrm{eV}$ (i.e. $0.55+0.37+0.68=1.6 \mathrm{eV}$ ). And this can be interpreted that the band gap consists of a recombination centre at $0.37 \mathrm{eV}$ above the valence band edge and a thermal excitation level (trap level) at $0.55 \mathrm{eV}$ below the conduction band edge. And the excited charge carriers are thermo-optically excited to the trap level which is $1.05 \mathrm{eV}$ above the valence band edge from which the charge carriers are further thermally excited into conduction band from a depth of $0.55 \mathrm{eV}$ as shown in band model diagram (figure 5).

\section{Acknowledgements}

The authors express their thanks to Ms W Xue and Dr Goldsmith, Physics Department, Boston College, USA for providing the crystal; and to Dr Babu Varghese, RSIC, IIT, Madras for the help offered in crystallographic studies.

\section{References}

Aviksoo J and Reinot T 1992 Mol. Cryst. Liq. Cryst. 217147

Batley M and Lyons L E 1970 Aust. J. Chem. 232397

Boer K W 1990 Survey of semiconductor physics (New York: Reinhold)

Eley D D, Parfitt G D, Perry M J and Taysum D H 1953 Trans. Faraday Soc. 4979

Gutmann $\mathrm{F}$ and Lyons L E 1981 Organic semiconductors (Part A) (Malabar: Krieger)

Hall G G 1991 Molecular solid state physics (Berlin: Springer)

Le Blanc O H $1962 J$. Chem. Phys. 361082

Mette H and Pick H 1953 Z. Physik. 134566

Mort J and Pai D M 1976 Photoconductivity and related phenomena (New York: Elsevier)

Riehl N V 1955 Zh. Fiz. Khim. 6959 
Riehl N V 1957 Ann. Physik. 6-20 93

Silinsh E A 1980 Organic molecular crystals (Berlin: Springer)

Simon J and Andre J-J 1985 Molecular semiconductors (Berlin: Springer)

Welfrich W and Lipsett E R 1965 J. Chem. Phys. 434368

Xavier F P 1993 Optical and transport properties of phthalocyanine and related compounds (Ann Arbor: UMI)

Xavier F P and Goldsmith G J 1995 Bull. Mater. Sci. 18269

Xue W and Goldsmith G J 1992 Private Communication 